\title{
A new version of the CNRM Chemistry-Climate Model, CNRM-CCM : description and improvements from the CCMVal-2 simulations - Supplementary document
}

M. Michou ${ }^{1}$, D. Saint-Martin ${ }^{1}$, H. Teyssèdre ${ }^{1}$, A. Alias ${ }^{1}$, F. Karcher ${ }^{1}$, D. Olivié ${ }^{1, a}$, A. Voldoire $^{1}$, B. Josse ${ }^{1}$, V.-H. Peuch ${ }^{1}$, H. Clark ${ }^{2}$, J. N. Lee ${ }^{3}$, and F. Chéroux ${ }^{1}$

'GAME/CNRM (Météo-France, CNRS) Centre National de Recherches Météorologiques, Toulouse, France

${ }^{a}$ now at University of Oslo, Norway

'Laboratoire d'Aérologie, Université de Toulouse, CNRS, Toulouse, France

${ }^{3}$ Jet Propulsion Laboratory, California Institute of Technology, Pasadena, USA

Correspondence to: M. Michou, D. Saint-Martin, H. Teyssèdre (martine.michou@meteo.fr, david.saint-martin@meteo.fr, hubert.teyssedre@meteo.fr) 


\section{References}

Cionni I., V. Eyring, J. F. Lamarque, W. J. Randel, D. S. Stevenson, F. Wu, G. E. Bodeker, T. G. Shepherd, D. T. Shindell, and D. W. Waugh, Ozone database in support of CMIP5 simulations: results and corresponding radiative forcing, Atmos. Chem. Phys. Discuss., 11, 10875-10933, 2011, www.atmoschem-phys-discuss.net/11/10875/2011/ doi:10.5194/acpd-11-10875-2011

Taylor, K. E., Summarizing multiple aspects of model performance in a single diagram, J. Geophys. Res., 106(D7), 7183-7192, 2001. 

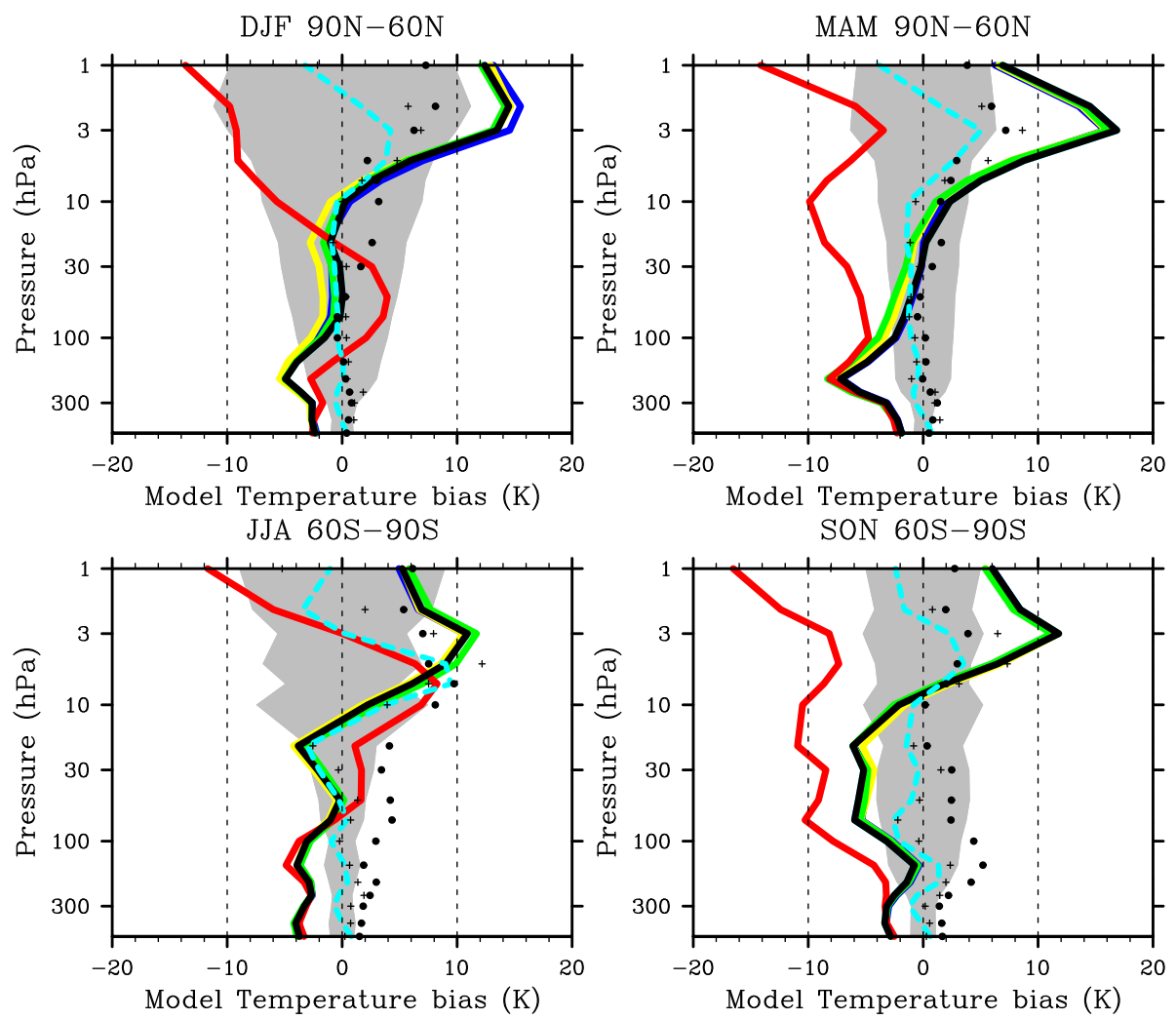

S 1. Temperature biases over two latitude ranges, $90^{\circ} \mathrm{N}-60^{\circ} \mathrm{N}$ (first row) and $60^{\circ} \mathrm{S}-90^{\circ} \mathrm{S}$ (second row), and two seasons, winter (left column) and spring (right column). Biases are relative to the ERA-40 19802001 monthly reanalysis, for CNRM-ACM (red line), and for five different "versions" of CNRM-CCM (see text, all other full lines, noting that two versions produced non separable lines), and for ERA-Interim (dashed cyan line). The grey area shows ERA- $40 \pm 1$ standard deviation. 
(a) 03 T7N MAR

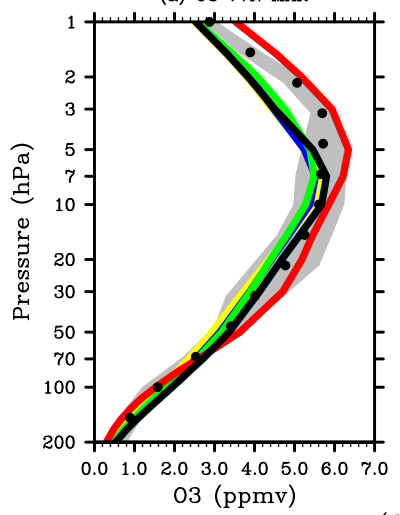

(b) 03 Equator MAR

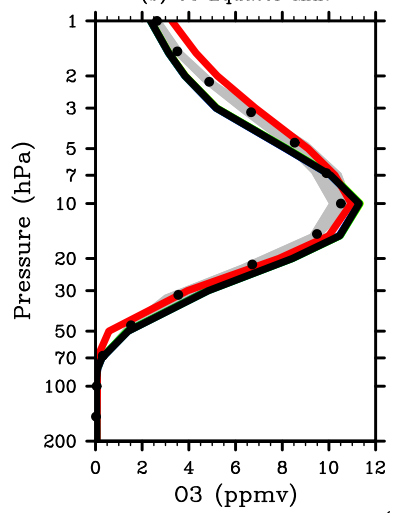

(c) $0377 \mathrm{~S}$ OCT

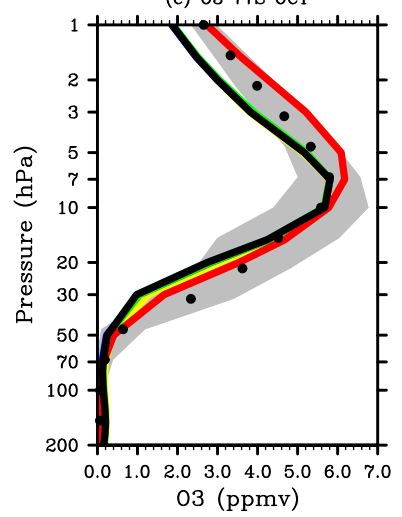

(d) $0350 \mathrm{hPa}$ MAR

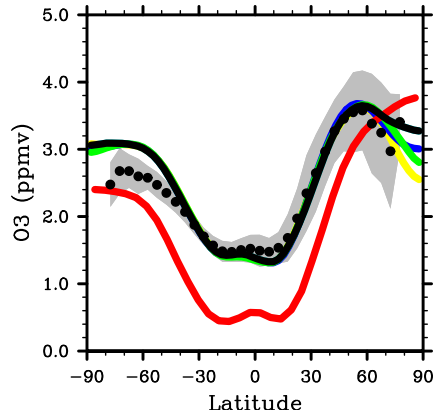

(e) $0350 \mathrm{hPa}$ OCT

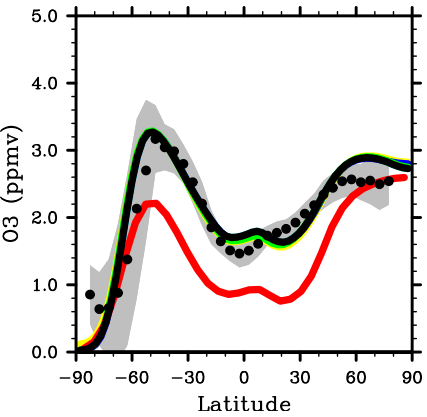

S 2. Climatological (1992-2001) zonal-mean $\mathrm{O}_{3}$ mixing (ppmv), for HALOE observations (black dots, with grey area showing $\pm 1 \sigma$ ), CNRM-ACM (red line), and for five different "versions" of CNRMCCM (all other full lines, noting that two versions produced non separable lines). Vertical profiles at (a) $77^{\circ} \mathrm{N}$ in March, (b) $0^{\circ} \mathrm{N}$ in March, and (c) $77^{\circ} \mathrm{S}$ in October. Zonal-means at $50 \mathrm{hPa}$ in (d) March and (e) October. 


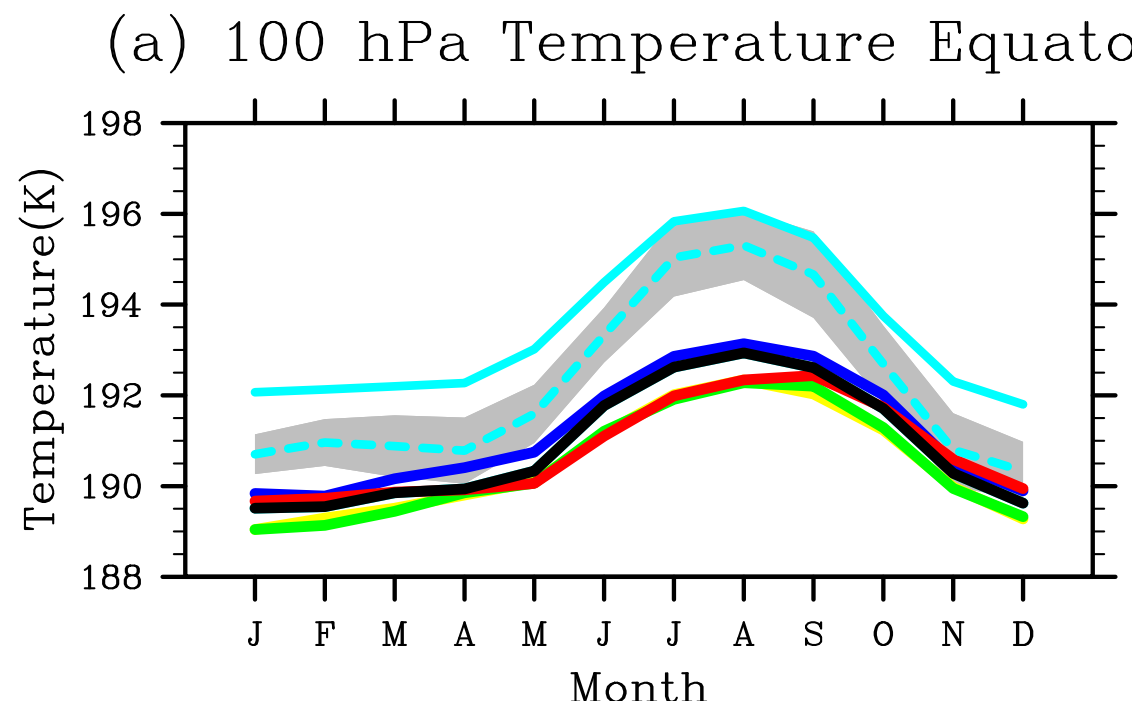

S 3. Zonal tropical climatologies of the temperature at Equator $100 \mathrm{hPa}$ with "observations", ERA-40 (cyan line), ERA-Interim (dashed cyan line, $\pm 1 \sigma$ 1989-2001). Models are also shown, CNRM-ACM (red line), and five different "versions" of CNRM-CCM (all other full lines, noting that two versions produced non separable lines). 

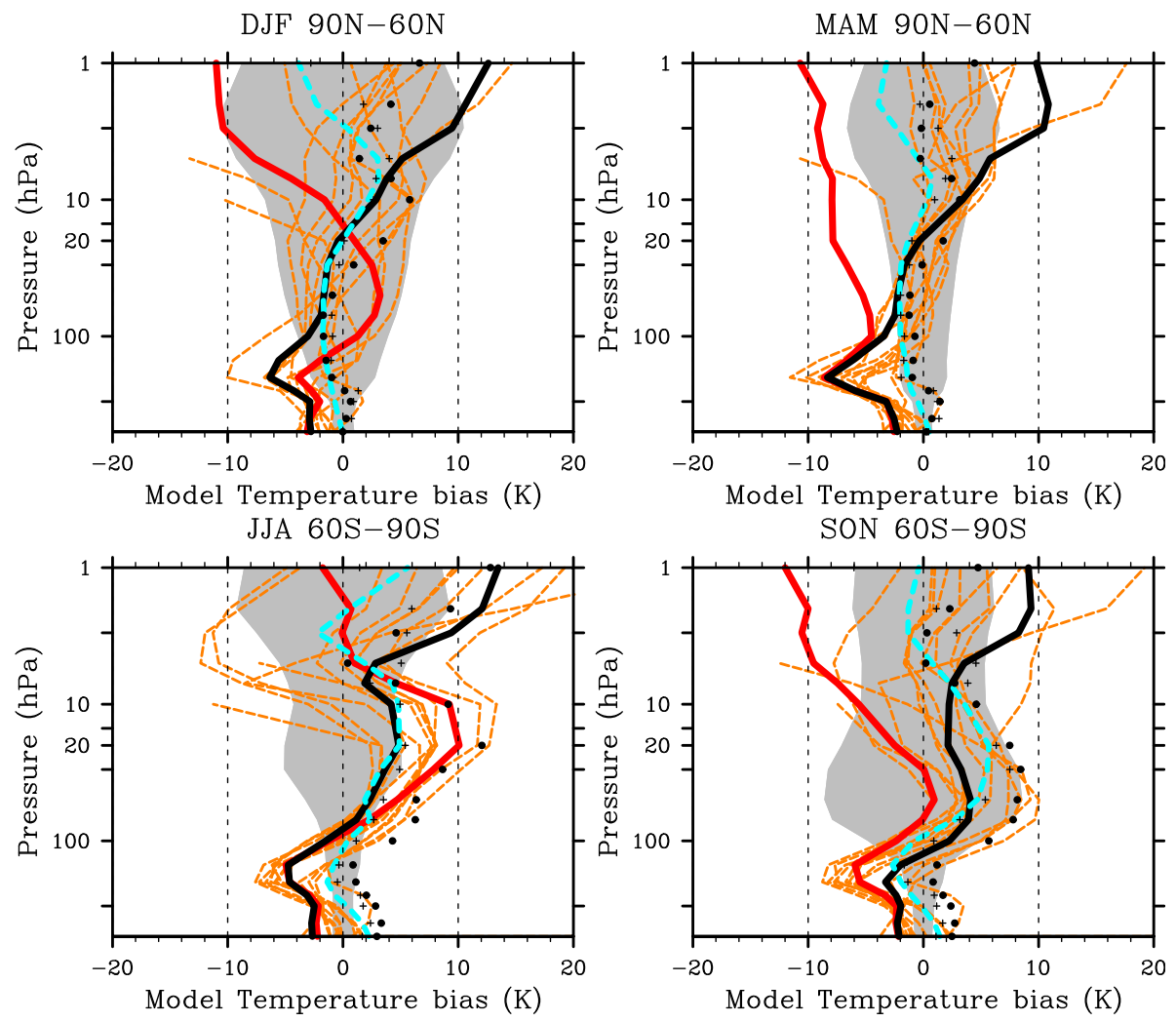

S 4. Temperature biases over two latitude ranges, $90 \mathrm{~N}-60 \mathrm{~N}$ (first row) and 60S-90S (second row), and two seasons, winter (left column) and spring (right column). Biases are relative to the ERA-40 19601980 monthly reanalysis, for CNRM-ACM (red line), CNRM-CCM (black line) and CCMVal-2 REFB1 models (dashed orange lines), and for ERA-Interim (dashed cyan line), NCEP (dots), and UKMO (crosses) reanalyses. The grey area shows ERA- $40 \pm 1$ standard deviation. 


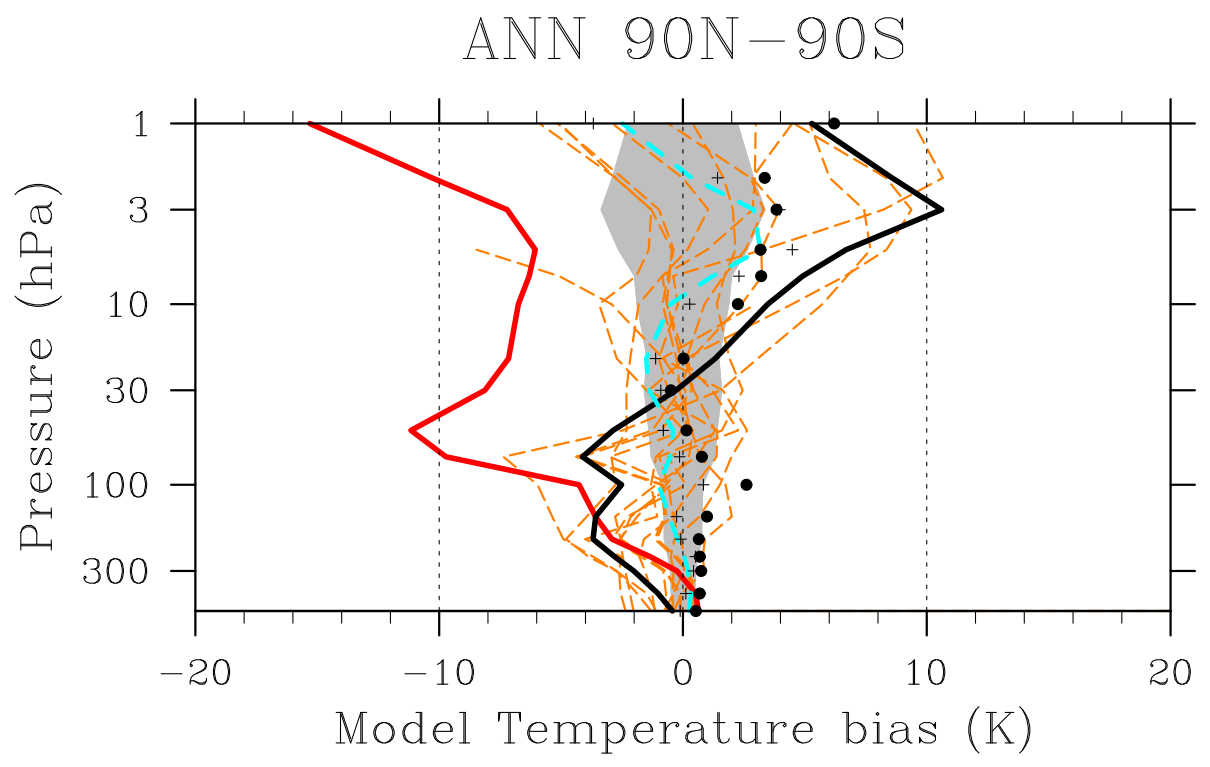

S 5. Annual temperature biases over $90 \mathrm{~N}-90 \mathrm{~S}$. Biases are relative to the ERA-40 1980-1999 monthly reanalysis, for CNRM-ACM (red line), CNRM-CCM (black line) and CCMVal-2 REF-B1 models (dashed orange lines), and for ERA-Interim (dashed cyan line), NCEP (dots), and UKMO (crosses) reanalyses. The grey area shows ERA- $40 \pm 1$ standard deviation. 

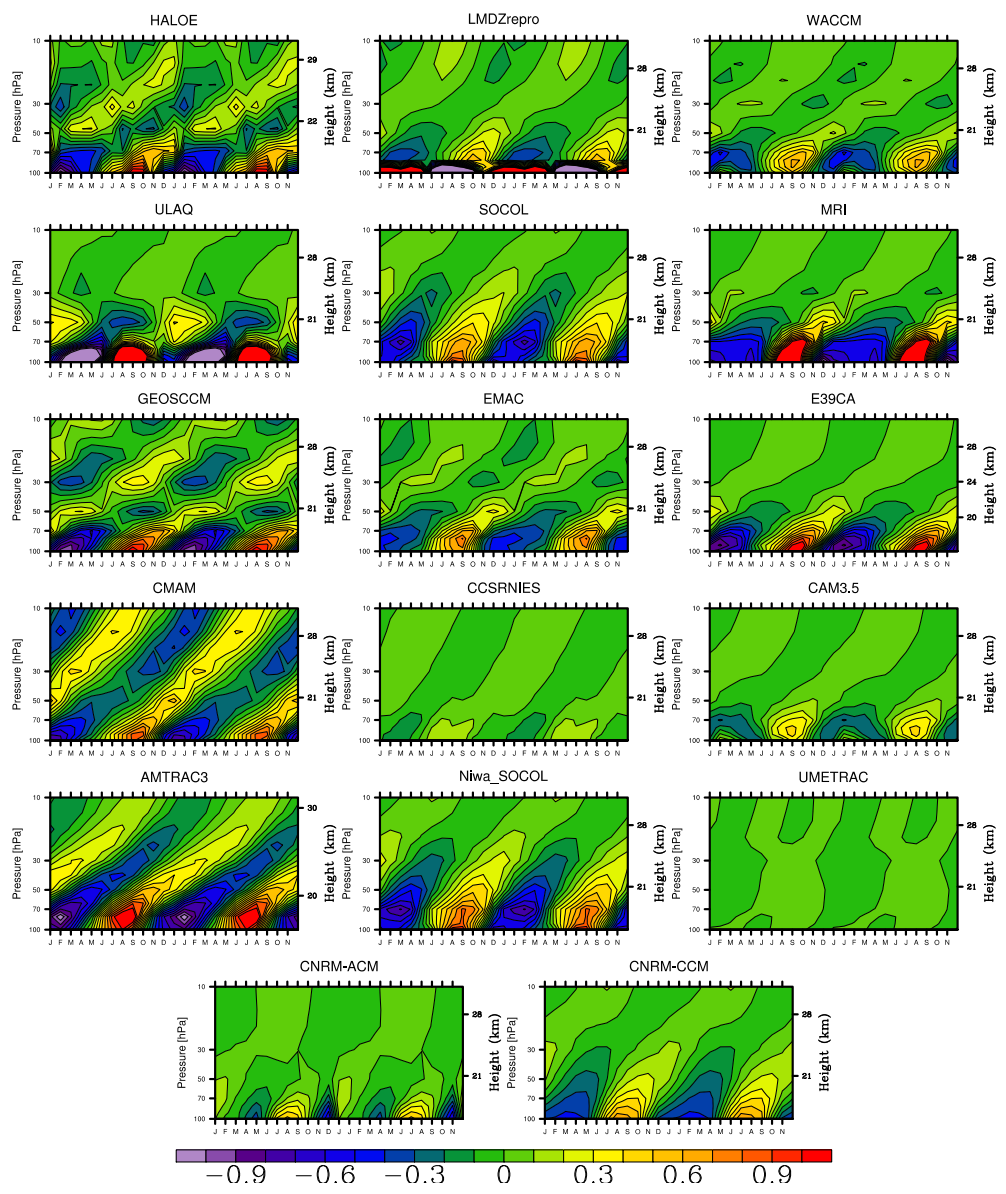

S 6. Deviations of the climatological monthly mean water vapor mixing ratio from the time mean ratio (1992-2001, averaged between 10S and 10N, ppmv), for HALOE observations and CCMVal-2 REF-B1 model simulations. CNRM-ACM and CNRM-CCM appear in the last row. Two identical cycles of the tape recorder are shown. 
(a) $\mathrm{CH} 4$ 77N MAR

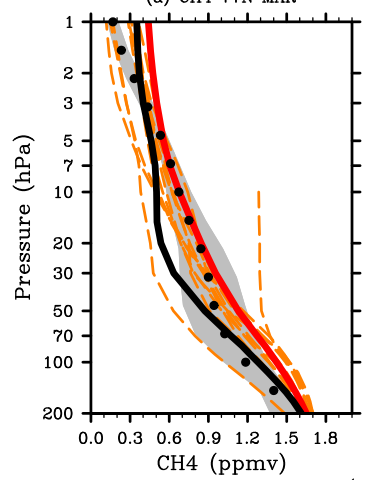

(b) CH4 Equator MAR

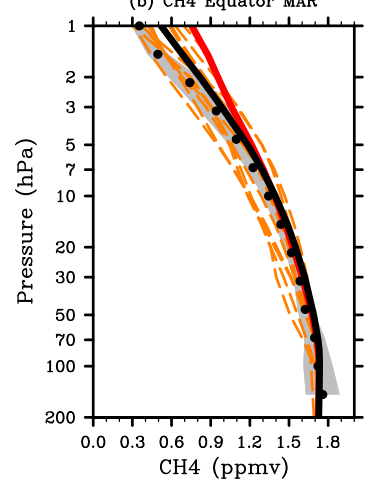

(c) $\mathrm{CH} 477 \mathrm{~S}$ OCT

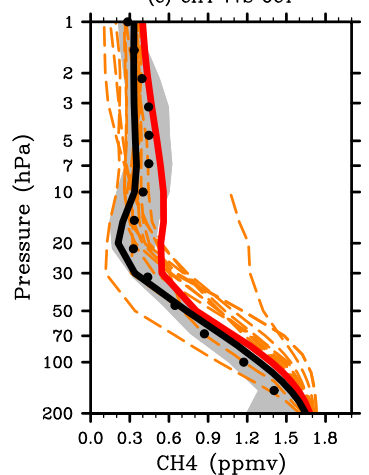

(d) $\mathrm{CH} 450 \mathrm{hPa}$ MAR
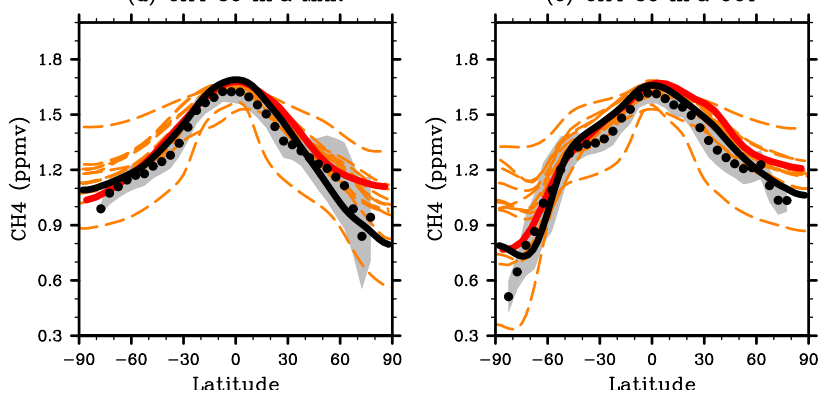

S 7. Climatological (1992-2001) zonal-mean $\mathrm{CH}_{4}$ mixing ratio (ppmv), for HALOE observations (black dots, with grey area showing $\pm 1 \sigma$ ), CNRM-ACM (red line), CNRM-CCM (black line), and CCMVal-2 REF-B1 models (dashed orange lines). Vertical profiles at (a) $77^{\circ} \mathrm{N}$ in March, (b) $0^{\circ} \mathrm{N}$ in March, and (c) $72^{\circ} \mathrm{S}$ in October. Zonal-means at $50 \mathrm{hPa}$ in (d) March and (e) October. 


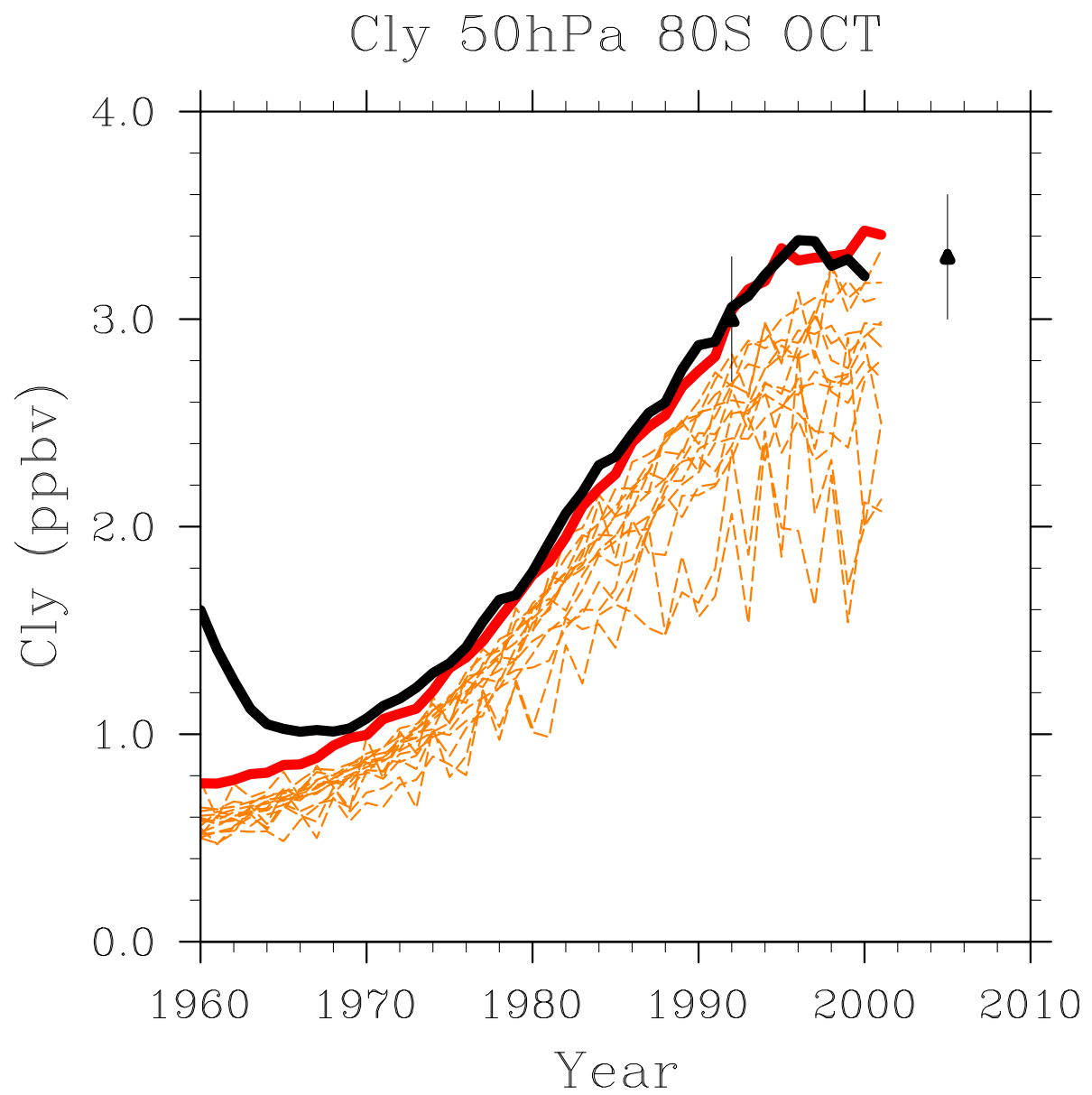

S 8. Time series of $\mathrm{Cl}_{\mathrm{y}}$ mixing ratios (ppbv) at $50 \mathrm{hPa}, 80 \mathrm{~S}$ in October over the REF-B1 period, with observations (diamonds, $\pm 1 \sigma$ ), CNRM-ACM (red line), CNRM-CCM (black line), and CCMVal-2 models outputs (orange dashed lines). 
DJF

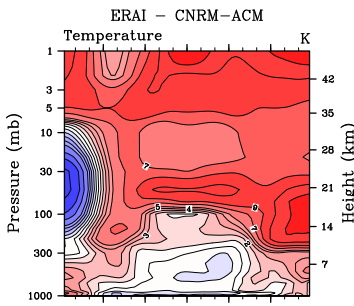

GoN
MIN $=-13.19$

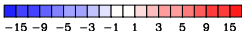

JJA

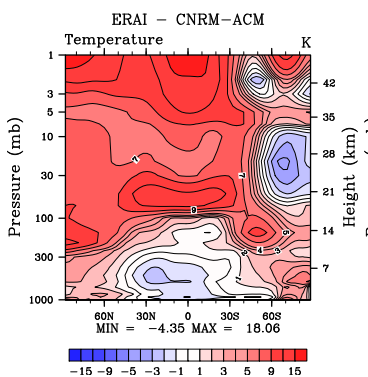

ERAI - CNRM-CCM

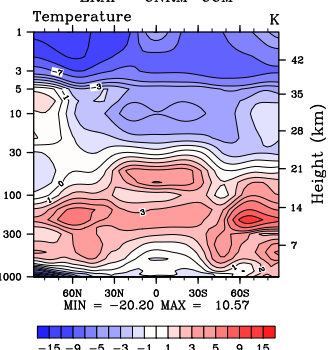

RAI - CNRM-CCM

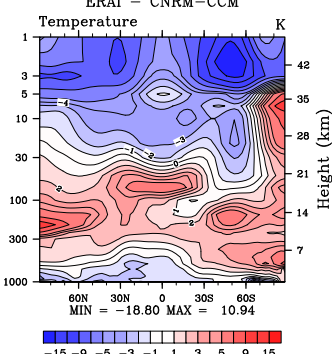

MAM
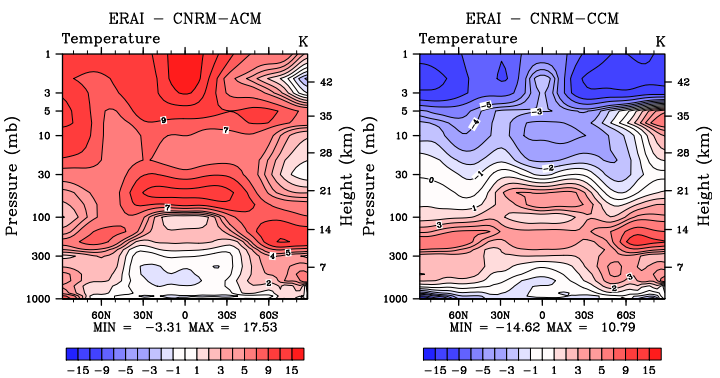

SON

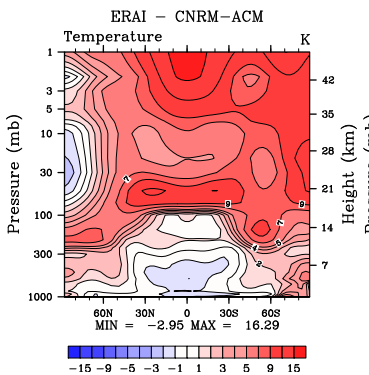

ERAI - CNRM-CCM

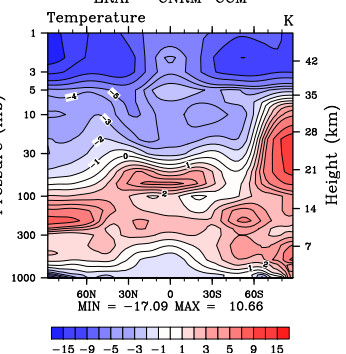

S 9. Latitude-pressure cross-sections of differences in temperature between ERA-Interim and CNRMACM (first and third columns), and between ERA-Interim and CNRM-CCM (second and fourth columns), for DJF and MAM (first row), and for JJA and SON (second row) (1989-2000 period). 
DJF

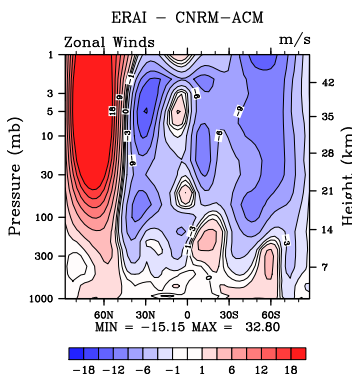

JJA

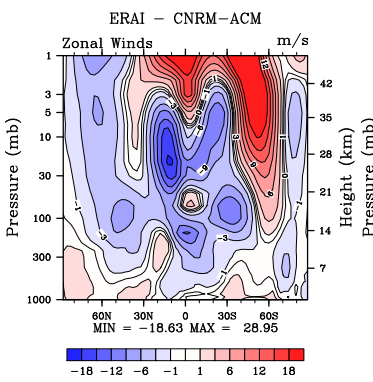

ERAI - CNRM-CCM

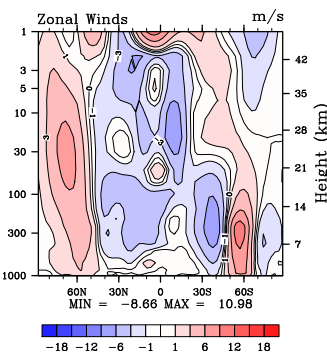

ERAI - CNRM-CCM

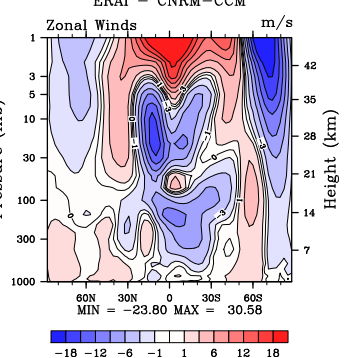

MAM
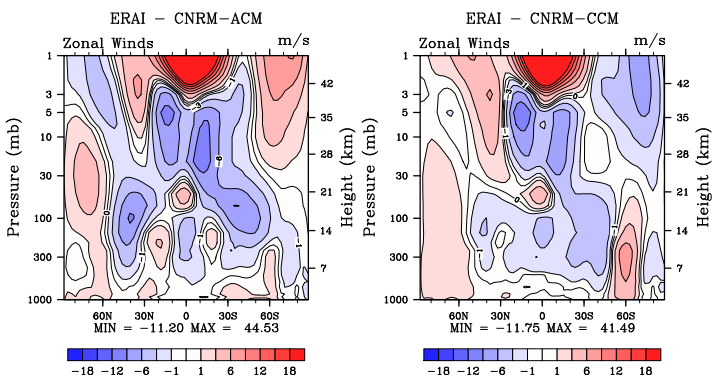

SON

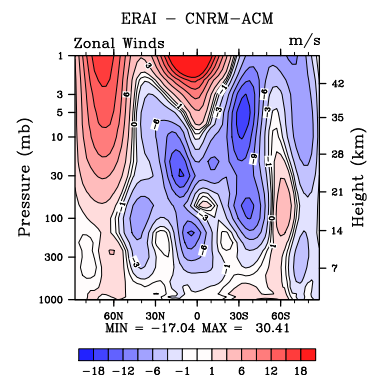

ERAI - CNRM-CCM

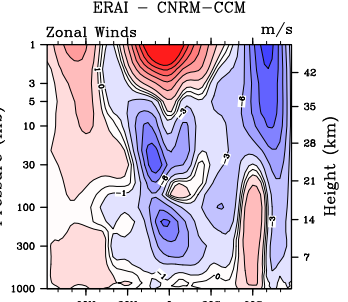

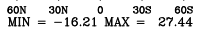

S 10. Latitude-pressure cross-sections of differences in zonal wind between ERA-Interim and CNRMACM (first and third columns), and between ERA-Interim and CNRM-CCM (second and fourth columns), for DJF and MAM (first row), and for JJA and SON (second row) (1989-2000 period). 

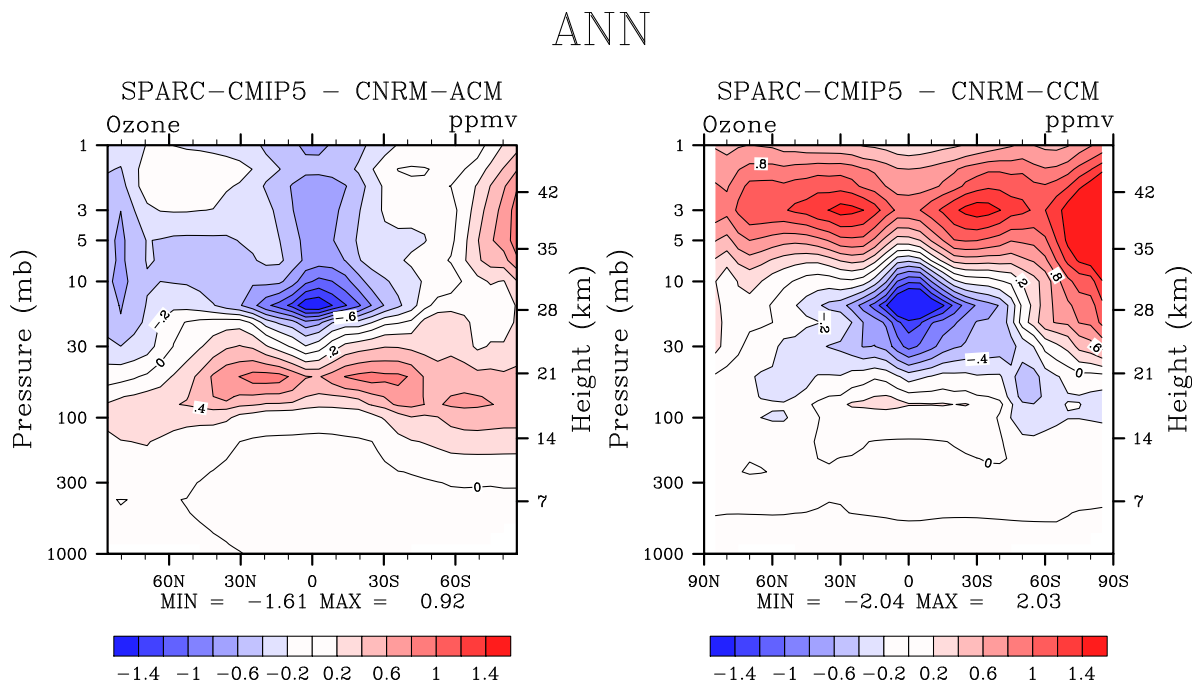

S 11. Latitude-pressure cross-section of differences in ozone (ppmv) between the AC\&C SPARC CMIP5 dataset (see Cionni et al. (2011)) and CNRM-ACM (left), or CNRM-CCM (right) (annual mean over 1990-1999). 

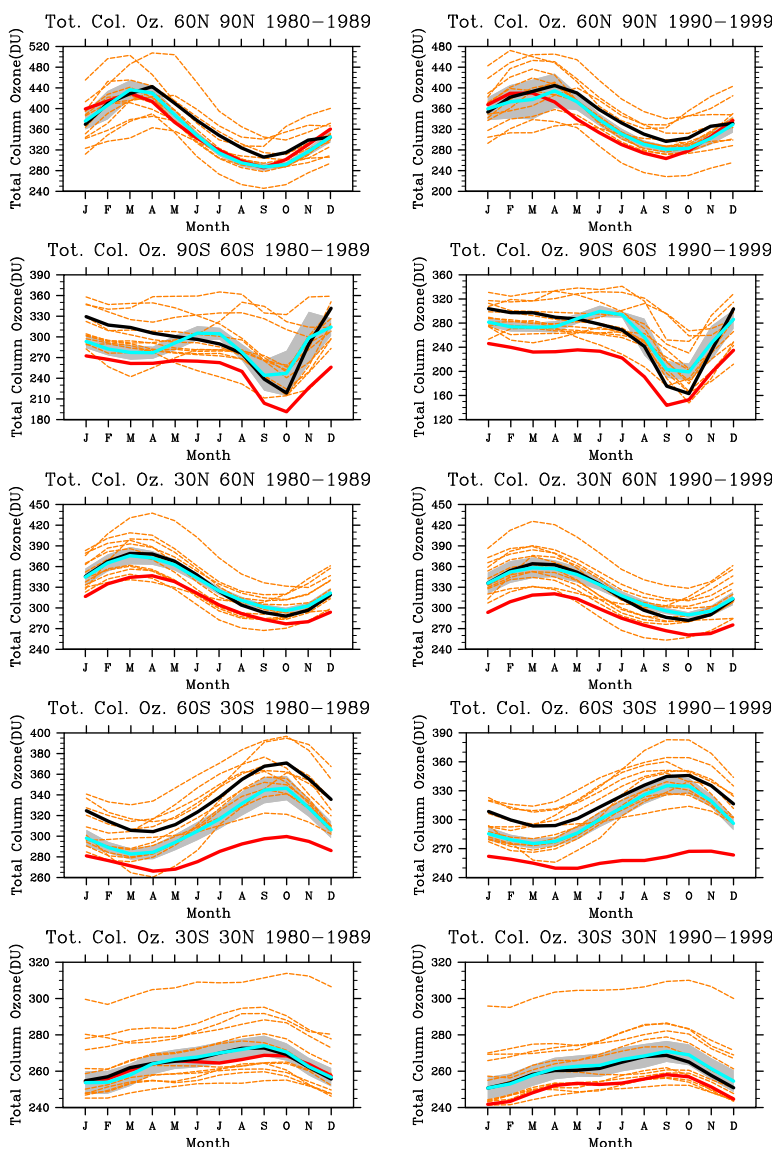

S 12. Zonal climatological (1980-1989 left column, 1990-1999 right column) total column ozone (DU) in various latitude bands, $60 \mathrm{~N}-90 \mathrm{~N}, 90 \mathrm{~S}-60 \mathrm{~S}, 30 \mathrm{~N}-60 \mathrm{~N}, 60 \mathrm{~S}-30 \mathrm{~S}$ and $30 \mathrm{~S}-30 \mathrm{~N}$, for NIWA BSv2.7 observations (cyan line) $\pm 1 \sigma$ (grey shading), CNRM-ACM (red line), CNRM-CCM (black line) and CCMVal-2 REB-B1 simulations (orange dashed lines). 


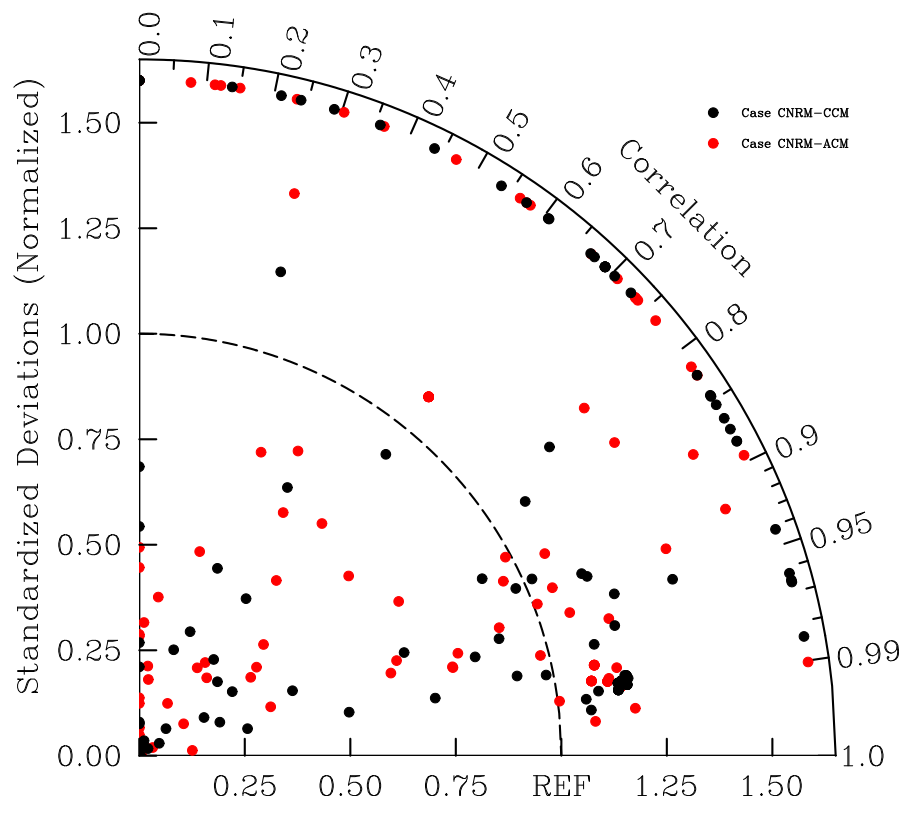

S 13. Taylor diagram (Taylor, 2001) of the diagnostics presented in the various paragraphs of the paper, red dots for CNRM-ACM, black dots for CNRM-CCM. 
Table 1. Results of the t-Tests conducted on each month of the annual cycle of a given chemical compound, hn1: $30^{\circ} \mathrm{N}-60^{\circ} \mathrm{N}$ at $1 \mathrm{hPa}$, hs $1: 30^{\circ} \mathrm{S}-60^{\circ} \mathrm{S}$ at $1 \mathrm{hPa}, \mathrm{hn} 50: 30^{\circ} \mathrm{N}-60^{\circ} \mathrm{N}$ at $50 \mathrm{hPa}$, hs50: $30^{\circ} \mathrm{S}-60^{\circ} \mathrm{S}$ at $50 \mathrm{hPa}$, trop1: $30^{\circ} \mathrm{S}-30^{\circ} \mathrm{N}$ at $1 \mathrm{hPa}$, trop50: $30^{\circ} \mathrm{S}-30^{\circ} \mathrm{N}$ at $50 \mathrm{hPa}$. A $\checkmark$ indicates where a significant difference $(\mathrm{p}<0.05)$ has been found.

\begin{tabular}{|c|c|c|c|c|c|c|c|c|c|c|c|c|}
\hline & Jan & Feb & Mar & Apr & May & Jun & Jul & Aug & Sep & Oct & Nov & Dec \\
\hline BrO-hn1 & & & & $\checkmark$ & $\checkmark$ & $\checkmark$ & $\checkmark$ & $\checkmark$ & & & & \\
\hline BrO-hn50 & $\checkmark$ & $\checkmark$ & $\checkmark$ & $\checkmark$ & $\checkmark$ & $\checkmark$ & $\checkmark$ & $\checkmark$ & $\checkmark$ & & $\checkmark$ & $\checkmark$ \\
\hline BrO-hs1 & $\checkmark$ & $\checkmark$ & & & & & $\checkmark$ & & & $\checkmark$ & $\checkmark$ & $\checkmark$ \\
\hline BrO-hs50 & $\checkmark$ & $\checkmark$ & $\checkmark$ & $\checkmark$ & & $\checkmark$ & $\checkmark$ & $\checkmark$ & & & & $\checkmark$ \\
\hline BrO-trop1 & $\checkmark$ & $\checkmark$ & $\checkmark$ & $\checkmark$ & $\checkmark$ & $\checkmark$ & $\checkmark$ & $\checkmark$ & $\checkmark$ & $\checkmark$ & $\checkmark$ & $\checkmark$ \\
\hline BrO-trop50 & $\checkmark$ & $\checkmark$ & $\checkmark$ & $\checkmark$ & $\checkmark$ & $\checkmark$ & $\checkmark$ & $\checkmark$ & $\checkmark$ & $\checkmark$ & $\checkmark$ & $\checkmark$ \\
\hline CH4-hn1 & $\checkmark$ & $\checkmark$ & $\checkmark$ & $\checkmark$ & $\checkmark$ & $\checkmark$ & $\checkmark$ & $\checkmark$ & $\checkmark$ & $\checkmark$ & $\checkmark$ & $\checkmark$ \\
\hline CH4-hn1 & $\checkmark$ & $\checkmark$ & $\checkmark$ & $\checkmark$ & $\checkmark$ & $\checkmark$ & $\checkmark$ & $\checkmark$ & $\checkmark$ & $\checkmark$ & $\checkmark$ & $\checkmark$ \\
\hline CH4-hn50 & $\checkmark$ & $\checkmark$ & $\checkmark$ & $\checkmark$ & $\checkmark$ & $\checkmark$ & $\checkmark$ & $\checkmark$ & $\checkmark$ & $\checkmark$ & $\checkmark$ & $\checkmark$ \\
\hline CH4-hn50 & $\checkmark$ & $\checkmark$ & $\checkmark$ & $\checkmark$ & $\checkmark$ & $\checkmark$ & $\checkmark$ & $\checkmark$ & $\checkmark$ & $\checkmark$ & $\checkmark$ & $\checkmark$ \\
\hline CH4-hs1 & $\checkmark$ & $\checkmark$ & $\checkmark$ & $\checkmark$ & $\checkmark$ & $\checkmark$ & $\checkmark$ & $\checkmark$ & $\checkmark$ & $\checkmark$ & $\checkmark$ & $\checkmark$ \\
\hline CH4-hs1 & $\checkmark$ & $\checkmark$ & $\checkmark$ & $\checkmark$ & $\checkmark$ & $\checkmark$ & $\checkmark$ & $\checkmark$ & $\checkmark$ & $\checkmark$ & $\checkmark$ & $\checkmark$ \\
\hline CH4-hs50 & $\checkmark$ & $\checkmark$ & $\checkmark$ & $\checkmark$ & $\checkmark$ & $\checkmark$ & $\checkmark$ & $\checkmark$ & $\checkmark$ & $\checkmark$ & $\checkmark$ & $\checkmark$ \\
\hline CH4-hs50 & $\checkmark$ & $\checkmark$ & $\checkmark$ & $\checkmark$ & $\checkmark$ & $\checkmark$ & $\checkmark$ & $\checkmark$ & $\checkmark$ & $\checkmark$ & $\checkmark$ & $\checkmark$ \\
\hline CH4-trop1 & $\checkmark$ & $\checkmark$ & $\checkmark$ & $\checkmark$ & $\checkmark$ & $\checkmark$ & $\checkmark$ & $\checkmark$ & $\checkmark$ & $\checkmark$ & $\checkmark$ & $\checkmark$ \\
\hline CH4-trop1 & $\checkmark$ & $\checkmark$ & $\checkmark$ & $\checkmark$ & $\checkmark$ & $\checkmark$ & $\checkmark$ & $\checkmark$ & $\checkmark$ & $\checkmark$ & $\checkmark$ & $\checkmark$ \\
\hline CH4-trop50 & $\checkmark$ & $\checkmark$ & $\checkmark$ & $\checkmark$ & $\checkmark$ & $\checkmark$ & & & & & $\checkmark$ & $\checkmark$ \\
\hline CH4-trop50 & $\checkmark$ & $\checkmark$ & $\checkmark$ & $\checkmark$ & $\checkmark$ & $\checkmark$ & & & & & $\checkmark$ & $\checkmark$ \\
\hline ClONO2-hn1 & $\checkmark$ & & & $\checkmark$ & $\checkmark$ & $\checkmark$ & $\checkmark$ & $\checkmark$ & $\checkmark$ & & $\checkmark$ & $\checkmark$ \\
\hline ClONO2-hn50 & $\checkmark$ & $\checkmark$ & & $\checkmark$ & $\checkmark$ & $\checkmark$ & $\checkmark$ & $\checkmark$ & $\checkmark$ & $\checkmark$ & $\checkmark$ & $\checkmark$ \\
\hline ClONO2-hs1 & $\checkmark$ & $\checkmark$ & & & $\checkmark$ & $\checkmark$ & & & $\checkmark$ & $\checkmark$ & $\checkmark$ & $\checkmark$ \\
\hline ClONO2-hs50 & $\checkmark$ & $\checkmark$ & $\checkmark$ & $\checkmark$ & $\checkmark$ & $\checkmark$ & & $\checkmark$ & $\checkmark$ & $\checkmark$ & $\checkmark$ & $\checkmark$ \\
\hline ClONO2-trop1 & $\checkmark$ & $\checkmark$ & $\checkmark$ & $\checkmark$ & $\checkmark$ & $\checkmark$ & $\checkmark$ & $\checkmark$ & $\checkmark$ & $\checkmark$ & $\checkmark$ & $\checkmark$ \\
\hline ClONO2-trop50 & $\checkmark$ & $\checkmark$ & $\checkmark$ & & & & & & & & $\checkmark$ & $\checkmark$ \\
\hline
\end{tabular}


Table 2. Same as Table 1, with hn100: $40^{\circ} \mathrm{N}-60^{\circ} \mathrm{N}$ at $100 \mathrm{hPa}$, hs $100: 40^{\circ} \mathrm{S}-60^{\circ} \mathrm{S}$ at $100 \mathrm{hPa}$, hn200: $40^{\circ} \mathrm{N}-60^{\circ} \mathrm{N}$ at $200 \mathrm{hPa}$, hs $200: 40^{\circ} \mathrm{S}-60^{\circ} \mathrm{S}$ at $200 \mathrm{hPa}$.

\begin{tabular}{|c|c|c|c|c|c|c|c|c|c|c|c|c|}
\hline & Jan & Feb & Mar & Apr & May & Jun & Jul & Aug & Sep & Oct & Nov & Dec \\
\hline CO-hn1 & $\checkmark$ & $\checkmark$ & & & & & & $\checkmark$ & $\checkmark$ & $\checkmark$ & $\checkmark$ & $\checkmark$ \\
\hline CO-hn50 & $\checkmark$ & $\checkmark$ & $\checkmark$ & $\checkmark$ & $\checkmark$ & $\checkmark$ & $\checkmark$ & $\checkmark$ & $\checkmark$ & $\checkmark$ & $\checkmark$ & $\checkmark$ \\
\hline CO-hs1 & $\checkmark$ & $\checkmark$ & $\checkmark$ & $\checkmark$ & & $\checkmark$ & $\checkmark$ & & & $\checkmark$ & & \\
\hline CO-hs50 & $\checkmark$ & $\checkmark$ & $\checkmark$ & $\checkmark$ & $\checkmark$ & $\checkmark$ & $\checkmark$ & $\checkmark$ & $\checkmark$ & $\checkmark$ & $\checkmark$ & $\checkmark$ \\
\hline CO-trop1 & $\checkmark$ & $\checkmark$ & $\checkmark$ & $\checkmark$ & $\checkmark$ & $\checkmark$ & $\checkmark$ & $\checkmark$ & $\checkmark$ & $\checkmark$ & $\checkmark$ & $\checkmark$ \\
\hline \multicolumn{13}{|l|}{ CO-trop50 } \\
\hline \multicolumn{13}{|l|}{$\mathrm{H} 2 \mathrm{O}$ Eq $100 \mathrm{hPa}$} \\
\hline \multicolumn{13}{|l|}{$\mathrm{H} 2 \mathrm{O} 20 \mathrm{~S} 20 \mathrm{~N} 80 \mathrm{hPa}$} \\
\hline H2O-hn100 & & $\checkmark$ & $\checkmark$ & $\checkmark$ & & & & & & & & \\
\hline H2O-hn1 & $\checkmark$ & $\checkmark$ & $\checkmark$ & $\checkmark$ & $\checkmark$ & $\checkmark$ & $\checkmark$ & $\checkmark$ & $\checkmark$ & $\checkmark$ & $\checkmark$ & $\checkmark$ \\
\hline H2O-hn200 & $\checkmark$ & $\checkmark$ & $\checkmark$ & & $\checkmark$ & $\checkmark$ & $\checkmark$ & & $\checkmark$ & $\checkmark$ & $\checkmark$ & $\checkmark$ \\
\hline H2O-hn50 & $\checkmark$ & $\checkmark$ & $\checkmark$ & $\checkmark$ & $\checkmark$ & $\checkmark$ & $\checkmark$ & $\checkmark$ & $\checkmark$ & $\checkmark$ & $\checkmark$ & $\checkmark$ \\
\hline \multicolumn{13}{|l|}{ H2O-hs 100} \\
\hline H2O-hs1 & $\checkmark$ & $\checkmark$ & $\checkmark$ & $\checkmark$ & $\checkmark$ & $\checkmark$ & $\checkmark$ & $\checkmark$ & $\checkmark$ & $\checkmark$ & $\checkmark$ & $\checkmark$ \\
\hline H2O-hs200 & $\checkmark$ & $\checkmark$ & $\checkmark$ & $\checkmark$ & $\checkmark$ & & & & & $\checkmark$ & $\checkmark$ & $\checkmark$ \\
\hline H2O-hs50 & $\checkmark$ & $\checkmark$ & $\checkmark$ & $\checkmark$ & $\checkmark$ & $\checkmark$ & $\checkmark$ & $\checkmark$ & $\checkmark$ & $\checkmark$ & $\checkmark$ & $\checkmark$ \\
\hline H2O-trop1 & $\checkmark$ & $\checkmark$ & $\checkmark$ & $\checkmark$ & $\checkmark$ & $\checkmark$ & $\checkmark$ & $\checkmark$ & $\checkmark$ & $\checkmark$ & $\checkmark$ & $\checkmark$ \\
\hline \multicolumn{13}{|l|}{ H2O-trop50 } \\
\hline HCl-hn1 & $\checkmark$ & $\checkmark$ & $\checkmark$ & $\checkmark$ & $\checkmark$ & $\checkmark$ & $\checkmark$ & $\checkmark$ & $\checkmark$ & $\checkmark$ & $\checkmark$ & $\checkmark$ \\
\hline HCl-hn50 & $\checkmark$ & $\checkmark$ & & & $\checkmark$ & $\checkmark$ & $\checkmark$ & $\checkmark$ & $\checkmark$ & $\checkmark$ & $\checkmark$ & \\
\hline HCl-hs1 & $\checkmark$ & $\checkmark$ & $\checkmark$ & $\checkmark$ & $\checkmark$ & $\checkmark$ & $\checkmark$ & $\checkmark$ & $\checkmark$ & $\checkmark$ & $\checkmark$ & $\checkmark$ \\
\hline HCl-hs50 & $\checkmark$ & $\checkmark$ & $\checkmark$ & $\checkmark$ & $\checkmark$ & $\checkmark$ & $\checkmark$ & $\checkmark$ & $\checkmark$ & $\checkmark$ & $\checkmark$ & $\checkmark$ \\
\hline HCl-trop1 & $\checkmark$ & $\checkmark$ & $\checkmark$ & $\checkmark$ & $\checkmark$ & $\checkmark$ & $\checkmark$ & $\checkmark$ & $\checkmark$ & $\checkmark$ & $\checkmark$ & $\checkmark$ \\
\hline \multicolumn{13}{|l|}{ HCl-trop50 } \\
\hline O3 20S20N $80 \mathrm{hPa}$ & $\checkmark$ & $\checkmark$ & $\checkmark$ & $\checkmark$ & $\checkmark$ & $\checkmark$ & & & $\checkmark$ & $\checkmark$ & $\checkmark$ & $\checkmark$ \\
\hline Temp Eq 100hPa & & & & & & & & & & & & \\
\hline
\end{tabular}


Table 3. Same as Tables 1 and 2.

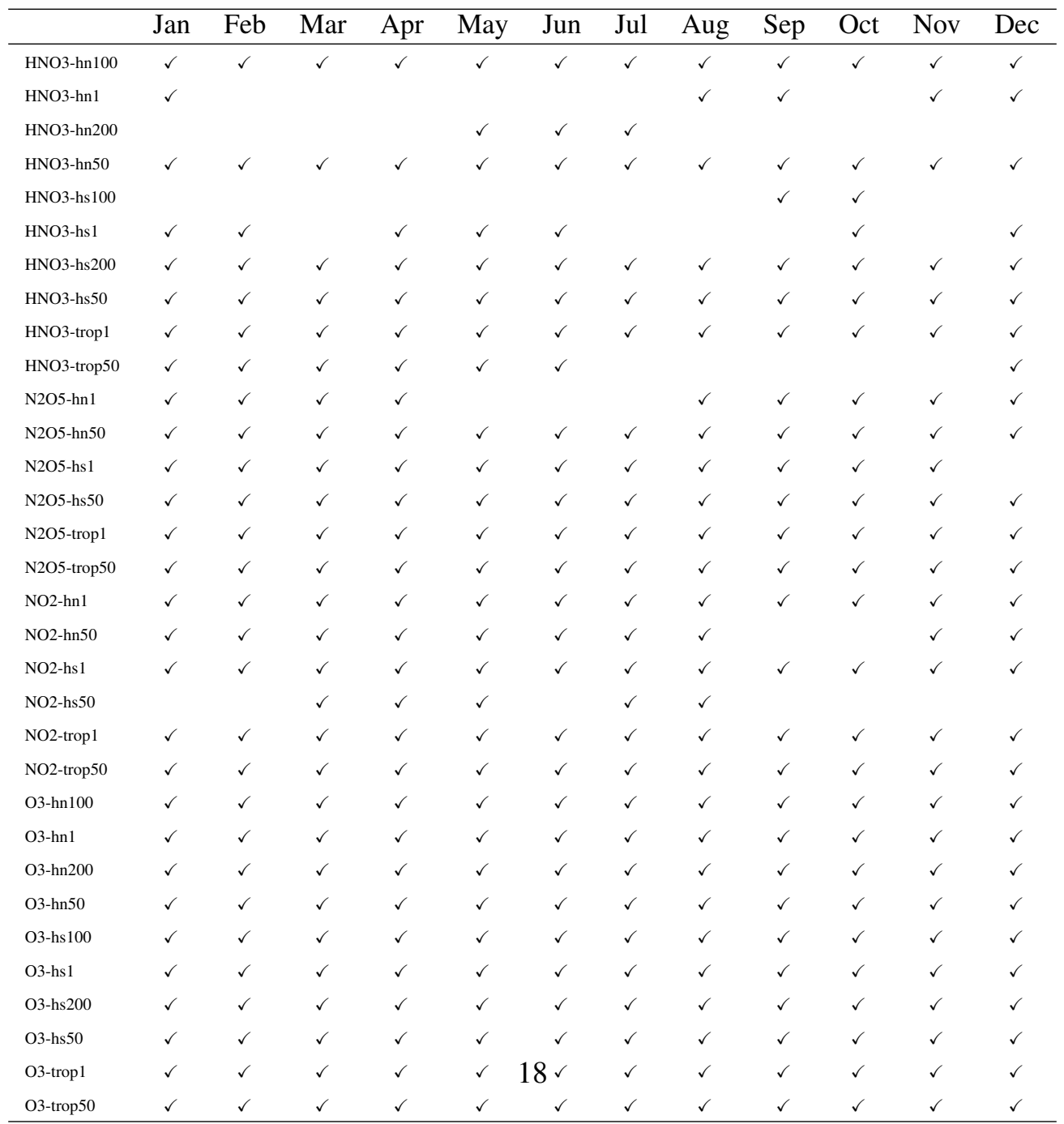


Table 4. Results of the t-Tests conducted on each level of the vertical profiles of a given chemical compound, for the months and latitudes of the diagnostics (see text and figures of the paper). A $\checkmark$ indicates where a significant difference $(\mathrm{p}<0.05)$ has been found.

\begin{tabular}{lllllllllllll}
\hline Diag/Level (hPa) & 1 & 2 & 3 & 5 & 7 & 10 & 20 & 30 & 50 & 70 & 100 & 200 \\
\hline CH4 Equa MAR & $\checkmark$ & $\checkmark$ & $\checkmark$ & $\checkmark$ & & & $\checkmark$ & $\checkmark$ & $\checkmark$ & $\checkmark$ & $\checkmark$ & $\checkmark$ \\
СH4 775 OCT & $\checkmark$ & $\checkmark$ & $\checkmark$ & $\checkmark$ & $\checkmark$ & $\checkmark$ & $\checkmark$ & $\checkmark$ & $\checkmark$ & $\checkmark$ & $\checkmark$ & $\checkmark$ \\
CH4 77N MAR & $\checkmark$ & $\checkmark$ & $\checkmark$ & $\checkmark$ & $\checkmark$ & $\checkmark$ & $\checkmark$ & $\checkmark$ & $\checkmark$ & $\checkmark$ & $\checkmark$ & $\checkmark$ \\
H20 Equa MAR & $\checkmark$ & $\checkmark$ & $\checkmark$ & $\checkmark$ & $\checkmark$ & $\checkmark$ & & & & & & $\checkmark$ \\
H2O 775 OCT & $\checkmark$ & $\checkmark$ & $\checkmark$ & $\checkmark$ & $\checkmark$ & $\checkmark$ & $\checkmark$ & & & & $\checkmark$ & $\checkmark$ \\
H20 77N MAR & $\checkmark$ & $\checkmark$ & $\checkmark$ & $\checkmark$ & $\checkmark$ & $\checkmark$ & $\checkmark$ & $\checkmark$ & $\checkmark$ & $\checkmark$ & $\checkmark$ & \\
HC1 Equa APR & $\checkmark$ & & & $\checkmark$ & $\checkmark$ & $\checkmark$ & $\checkmark$ & $\checkmark$ & $\checkmark$ & $\checkmark$ & $\checkmark$ & $\checkmark$ \\
HC1 72S NOV & & $\checkmark$ & $\checkmark$ & $\checkmark$ & $\checkmark$ & & $\checkmark$ & $\checkmark$ & $\checkmark$ & $\checkmark$ & $\checkmark$ & $\checkmark$ \\
HC1 77N APR & & $\checkmark$ & $\checkmark$ & $\checkmark$ & & & $\checkmark$ & $\checkmark$ & & & $\checkmark$ & $\checkmark$ \\
О3 Equa MAR & $\checkmark$ & $\checkmark$ & $\checkmark$ & $\checkmark$ & $\checkmark$ & $\checkmark$ & $\checkmark$ & $\checkmark$ & $\checkmark$ & $\checkmark$ & $\checkmark$ & $\checkmark$ \\
О3 775 OCT & $\checkmark$ & $\checkmark$ & $\checkmark$ & $\checkmark$ & $\checkmark$ & & $\checkmark$ & $\checkmark$ & & & $\checkmark$ & $\checkmark$ \\
О377N MAR & $\checkmark$ & $\checkmark$ & $\checkmark$ & $\checkmark$ & $\checkmark$ & & $\checkmark$ & $\checkmark$ & & & $\checkmark$ & $\checkmark$ \\
\hline
\end{tabular}

